\title{
Convective dispersion without molecular diffusion
}

\author{
Kevin D. Dorfman and Howard Brenner* \\ Department of Chemical Engineering \\ Massachusetts Insitute of Technology \\ Cambridge, MA 02139-4307 USA
}

March 30, 2018

\begin{abstract}
A method-of-moments scheme is invoked to compute the asymptotic, long-time mean (or composite) velocity and dispersivity (effective diffusivity) of a two-state particle undergoing one-dimensional convectivediffusive motion accompanied by a reversible linear transition ("chemical reaction" or "change in phase") between these states. The instantaneous state-specific particle velocity is assumed to depend only upon the instantaneous state of the particle, and the transition between states is assumed to be governed by spatially-independent, first-order kinetics. Remarkably, even in the absence of molecular diffusion, the average transport of the "composite" particle exhibits gaussian diffusive behavior in the long-time limit, owing to the effectively stochastic nature of the overall transport phenomena induced by the interstate transition. The asymptotic results obtained are compared with numerical computations.
\end{abstract}

Keywords: Homogenization, Brownian motion, macrotransport theory, generalized Taylor dispersion

PACS: $05.40 . J c, 87.10 .+\mathrm{e}$

\section{Introduction}

Two-state models are often invoked to construct simple, easily analyzed transport models of otherwise overwhelmingly complex processes. Examples of such processes include chromatography [1,2], isomeric conversion of proteins in solution and in vivo calcium kinetics [3], and ratchet-driven molecular motor processes [4. In such models, it is typically assumed that the species of interest, either a single entity (dispersed in a passive solvent) or a continuum solute concentration field composed of a dilute collection of such non-interacting species (again dispersed in a passive solvent), undergo(es) a microscale, state-specific,

${ }^{*}$ Corresponding author. Address: 77 Massachusetts Ave., Room 66-564, Cambridge, MA 02139-4307 USA. Phone: 617-253-6687. Fax: 617-258-8224. Email: hbrenner@mit.edu. 
convective-diffusive transport process wherein the transport coefficients depend at time $t$ upon the "state" $(a, b)$ of the species at that time. The rate of interchange between states is assumed to be governed by first-order kinetics. Since the total species number density does not change in time, this kinetic process is equivalent in its consequences to a reversible first-order reaction or phase transition, $a \leftrightarrow b$, between states $a$ and $b$. In almost all cases of interest, one is not generally concerned with the detailed intrastate transport of the species in state $a$ or $b$ individually, but rather only with knowledge of the long-time, asymptotic aggregate transport of the composite $a-b$ species; that is, interest focuses only on the combined transport occurring in both states $a$ and $b$ for times sufficiently long such that the interstate reaction has effectively achieved global equilibrium (although the reaction may not be in local equilibrium at each point in space). Remarkably, this averaged aggregate transport is diffusive, even in the absence of molecular diffusion.

Many two-state models only feature the explicit incorporation of convection and a "reaction," without explicitly accounting for intrastate molecular diffusion. Consequently, it is tempting to say that these models constitute examples of "convective dispersion without molecular diffusion." However, the interstate transfer reaction often corresponds to a simple model for the solute's molecular diffusion, allowing it to sample two different velocity fields prevailing at different points in space, say, in two different phases. In an exactly-posed model [5], molecular diffusion transports the solute to the interphase boundary, whereupon an interstate mass transfer "reaction" occurs. Without the explicit incorporation of diffusion, a simplified (or rate limited) one-dimensional model [12] allows the solute to "jump" between phases in a kinetic manner. The dispersivity computed from such a "nondiffusive" model [2, which possesses a mathematical structure similar to the generic problem to be considered here, does indeed furnish a convective dispersivity which is explicitly independent of the molecular diffusivity. From a physical viewpoint, though, the convective dispersion may still be attributed to molecular diffusion, but at a finer-scale level of description than is represented by the coarse-scale transport equations adopted in our model. As such, it is not entirely correct to say that existing two-state chromatography models satisfy our claim of "convective dispersion without molecular diffusion."

Rather, our claim more closely corresponds to the case where the dispersion arising from the interstate reaction does not occur as a consequence of molecular diffusion involving solute molecules which randomly shuttle back and forth between two different velocity fields (phases) located at different points in space. To see how such a scenario arises, consider the examples of molecular motors and denaturing of double-stranded DNA. In the case of molecular motors [4, the species can exist in either a charged or uncharged state. When charged, the particle undergoes convective transport due to its interaction with a charged potential field, the latter referred to as the "track." During the course of its convective motion, the particle may also interact biophysically with the surrounding medium (say, by incorporating ATP). The latter interactions neu- 
tralize the particle and arrest its convective motion. ${ }^{1}$ The overall biophysical process is cyclical, so the particle may again become uncharged, enabling the transition between charged and uncharged states to be modeled as a reversible chemical reaction. Though the particle itself also undergoes Brownian motion, the transitions between the charged and uncharged states depend only upon the chemical interactions of the particle with the surrounding medium, not the spatial location of the particle.

Another example of a non-diffusive change in state involves the partial denaturing of double-stranded DNA. In such circumstances, the two associated strands become locally separated over a short range of base pairs, owing to an elevated temperature (or an equivalent concentration of denaturant) exceeding the local melting temperature. The native-denatured transition is primarily attributed to a chemical interaction between the base pairs (and the denaturant, if present), rather than to molecular diffusion. The native and denatured states possess distinctly different electrophoretic mobilities, and it is these differences which have been exploited in the context of numerous electrophoretic schemes [7, 8] for the detection of disease-causing mutations. Inasmuch as the mutation detection relies upon interpreting the chromatographic bands, it would be useful to have a greater understanding of the role of fluctuations between the states (which could be related to, say, fluctuations in the effective temperature of the medium) upon the overall chromatographic process. In both the motor and denatured DNA cases, the change in the solute state, and thus the solute velocity, arises from a chemical interaction with the medium, not from a conventional molecular diffusion process transporting the solute to a new point in space. Consequently, the convective dispersion process arising here would persist in the absence of molecular diffusion.

As a prelude to a much more general analysis, the main point of this paper is illustrated by considering the following elementary non-diffusive system:

$$
\begin{aligned}
\frac{\partial P_{a}}{\partial t}+U \frac{\partial P_{a}}{\partial x}+k\left(P_{a}-P_{b}\right) & =\frac{1}{2} \delta(x) \delta(t), \\
\frac{\partial P_{b}}{\partial t}-U \frac{\partial P_{b}}{\partial x}+k\left(P_{b}-P_{a}\right) & =\frac{1}{2} \delta(x) \delta(t),
\end{aligned}
$$

where $P_{\alpha}(x, t)$ is the conditional probability density of state $\alpha, U$ is the species velocity, $k$ is the interstate reaction rate, and $\delta$ is the Dirac delta function. Let $\hat{P}_{\alpha}(q, s)$ denote the Fourier-Laplace transform of $P_{\alpha}(x, t)$, with $q$ the Fourier variable and $s$ the Laplace variable. Transforming eqs. (1.1)-(1.2) into FourierLaplace space furnishes the following coupled set of algebraic equations:

$$
s \hat{P}_{a}+(i q U) \hat{P}_{a}+k\left(\hat{P}_{a}-\hat{P}_{b}\right)=\frac{1}{2}, \quad s \hat{P}_{b}-(i q U) \hat{P}_{b}+k\left(\hat{P}_{b}-\hat{P}_{a}\right)=\frac{1}{2} .
$$

\footnotetext{
${ }^{1}$ In more realistic models, the tracks possess a spatially-periodic, ratchet-like charge distribution. While modeling periodic systems necessitates a more elaborate moment-matching scheme than the one employed here, the existing macrotransport scheme 6 for single-state systems is readily extensible to two-states. The resulting calculations are more involved, but the final result reveals, as would be expected, that a similar contribution to the convective dispersion exists in the periodic case.
} 
Upon solving for $\hat{P}_{a}$ and $\hat{P}_{b}$ and forming their sum, the Fourier-Laplace transform of the total probability density adopts the form

$$
\hat{P}=\hat{P}_{a}+\hat{P}_{b}=\left(s+\frac{q^{2} U^{2}}{s+2 k}\right)^{-1} .
$$

For long times $t \gg 2 k$, corresponding here to $s \ll 2 k$, this becomes

$$
\hat{P} \approx\left(s+\frac{q^{2} U^{2}}{2 k}\right)^{-1} .
$$

The latter expression is readily inverted to yield the gaussian distribution,

$$
P \approx\left(4 \pi \bar{D}^{*} t\right)^{-1 / 2} \exp \left[-\frac{x^{2}}{4 \bar{D}^{*} t}\right]
$$

with an effective diffusion (dispersion) coefficient

$$
\bar{D}^{*}=\frac{U^{2}}{2 k}
$$

Moreover, the mean position of the composite particle remains at the initial position $x=0$. The latter property is equivalent in its consequences to a zero composite particle velocity, $\bar{U}^{*}=0$ [compare eq. (3.5)].

In conventional long-time average "macrotransport" analyses [6] of this type, the dispersivity $\bar{D}^{*}$ arises via the stochastic sampling of local velocity inhomogeneities by solute diffusion in the continuous local space. For example, in the classic case of Taylor dispersion 9, quantifying the global solute transport occurring in a Poiseuille flow within a long circular cylindrical tube, radial diffusion across the streamlines enables a solute molecule being transported axially by the Poiseuille flow to sample the fluid's transversely parabolic axial velocity profile innumerable times as it moves downstream, eventually attaining a stationary mean velocity $\bar{U}^{*}$ and dispersivity $\bar{D}^{*}$. In contrast, the source of the dispersion in our two-state problem resides in the interstate reaction, which allows the composite $a-b$ species to sample the discrete "state space." Nevertheless, dispersion arising from the sampling of different state-specific velocities is completely analogous to the dispersion which arises from so-called "local-space" velocity inhomogeneities, e.g. Poiseuille flow, in classical macrotransport theory [6]. The stochastic foundation of chemical reactions is well established [10, inasmuch as the reaction rate $k$ may be interpreted as reflecting the probability of a reaction occurring in the interval between times $t$ and $t+\delta t$. Moreover, whereas chemical reactions are considered to constitute purely deterministic processes in the full position-momentum space ("local space"), the process is nevertheless stochastic in the reduced space ("global space") employed in conventional kinetic models 11]. Likewise, the comparable reduction from transport in the distinct states $a$ and $b$ to transport in the composite $a-b$ state results in a stochastic (diffusive) composite transport process. 
In the present contribution, we investigate more thoroughly this surprising property of two-state systems. This is effected by computing, via the theory of macrotransport processes [6], the asymptotic, long-time mean velocity, $\bar{U}^{*}$, and dispersivity, $\bar{D}^{*}$, of a more general two-state system than that defined by eqs. (1.1)-(1.2). This scheme may be likened to a multiple-time scale analysis [12] of the phenomena, where the short-time, two-state behavior ultimately serves to determine the long-time, state-independent temporal behavior of the system as a whole. In a very different, non-reactive context than that considered here, moment-matching concepts have been used [5] 13] to consider systems where the different "states" correspond to the different physical phases (e.g. solid and liquid) through which a solute molecule can be transported at different velocities. In contrast with the present analysis, the latter analyses employed molecular diffusion to transport the solute to the interface across which the interstate mass transfer (change in state) occurs. Subsequently, Iosilevskii and Brenner [14] used eigenfunction expansions of the moments to develop a general macrotransport scheme for the analysis of reactive mixtures in an incompressible solvent flow, where molecular diffusion again played an important role in enabling the solute to sample the local space. Balakotaiah and Chang [2] used center-manifold techniques to examine problems in chromatography theory which are similar to those considered here, albeit with an implicit dependence upon molecular diffusion. The results of the latter correspond to a special case of the subsequent analysis, which, we believe, employs a more straightforward technique than is manifest in center manifold theory. ${ }^{2}$

\section{Generalized Problem Statement}

Consider the conditional probability density $P_{\alpha}\left(x, t \mid \phi_{\alpha}\right)(\alpha=a, b)$ that a noninteracting collection of particles exists in state $\alpha$ and are present at position $x$ at time $t$, given their initial impulsive introduction into the unbounded system $(-\infty<x<\infty)$ at $x=0$ and time $t=0$, with $\phi_{\alpha}$ the fraction of the particles initially in state $\alpha$. The two $\phi_{\alpha}$ are not independent parameters, since $\phi_{a}+$ $\phi_{b}=1$. If an effective or composite $a-b$ "particle" description is to exist, the final results for $\bar{U}^{*}$ and $\bar{D}^{*}$ quantifying the spatio-temporal transport of this composite "particle" must (and will) prove to be independent of the arbitrary choice of labels $a$ and $b$. Moreover, the macrotransport parameters $\bar{U}^{*}$ and $\bar{D}^{*}$ will further prove to be independent of the particle's initial arbitrary position $x=0$, as well as of the initial state fractions $\phi_{\alpha}$.

The spatio-temporal evolution of the conditional probability densities, $P_{a} \equiv$ $P_{a}\left(x, t \mid \phi_{a}\right)$ and $P_{b} \equiv P_{b}\left(x, t \mid \phi_{b}\right)$, are governed by the coupled set of convectiondiffusion-reaction equations,

$$
\frac{\partial P_{\alpha}}{\partial t}+U_{\alpha} \frac{\partial P_{\alpha}}{\partial x}-D_{\alpha} \frac{\partial^{2} P_{\alpha}}{\partial x^{2}}+k\left(K_{\alpha} P_{\alpha}-K_{\beta} P_{\beta}\right)=\phi_{\alpha} \delta(t) \delta(x),
$$

\footnotetext{
${ }^{2}$ It should be noted that the moment-matching scheme employed here is only valid for linear (first-order) reactions, whereas the center-manifold technique may be employed to analyze nonlinear transport processes as well 2 .
} 
valid for $\alpha=(a, b)(\alpha \neq \beta)$, with $\delta$ the Dirac delta function. Here, the constants $U_{\alpha}$ and $D_{\alpha}$ represent the state-specific velocity and molecular diffusivity of the particle. It is assumed that the latter parameters depend solely upon the state $(a, b)$ of the particle, being independent of position $x$, as well as of time $t$. While it is possible in the interest of simplicity to set the state diffusivities $D_{\alpha}$ to zero at the outset, we retain these terms in order to assess what will prove to be their relatively straightforward impact upon the averaged long-time transport process [cf. eqs. (3.8)-(3.9)].

In the above, the transition between states has been assumed to occur via first-order kinetics, with $k K_{a}$ representing the transition rate from state $a$ to $b$, and $k K_{b}$ the transition rate from $b$ to $a$, with all reaction parameters being positive: $\left(k, K_{a}, K_{b}\right)>0$. We employ the trio of parameters $k, K_{a}$ and $K_{b}$ (of which only two are independent, say $k K_{a}$ and $K \equiv K_{b} / K_{a}$ ), rather than a pair of reaction rates, say, $k_{a}=k K_{a}$ and $k_{b}=k K_{b}$, so as to permit a clear distinction to be made between the kinetic rate of interstate transfer, embodied in $k$, and the stationary (equilibrium) partitioning of states, embodied in $K_{b} / K_{a}$. Moreover, this egalitarian notational choice serves simultaneously to emphasize that the final results are independent from the arbitrary choice of labels $a$ and $b$.

This coupled set of partial differential equations can be solved, in principle, subject to the attenuation of the conditional probability densities and fluxes ${ }^{3}$ at infinity for all times $t>0$ :

$$
P_{\alpha}, J_{\alpha} \rightarrow 0 \quad \text { as } \quad|x| \rightarrow \infty .
$$

Summing eqs. (2.1) over $a$ and $b$, and subsequently integrating over the interval $(-\infty, \infty)$ furnishes the particle conservation relation,

$$
\int_{-\infty}^{\infty}\left(P_{a}+P_{b}\right) d x=1 \quad(t>0)
$$

which reflects the fact that each of the particles originally introduced into the system at time $t=0$ are conserved for all time, independently of the initial distributions, $\phi_{a}$ and $\phi_{b}$.

\section{Macrotransport Analysis}

In Appendix A, we use standard moment-matching techniques [6] to analyze the generalized problem for asymptotically long times, namely those satisfying the inequality

$$
t \gg\left[k\left(K_{a}+K_{b}\right)\right]^{-1}
$$

\footnotetext{
${ }^{3}$ When the $D_{\alpha}$ are non-zero, the $P_{\alpha}$ are expected to decay exponentially fast as $|x| \rightarrow \infty$, whence the flux attenuation condition in eq. 2.2 will be automatically satisfied. For the case where the $D_{\alpha}$ are identically zero, the governing equation 2.1 is hyperbolic, whereupon the probability density is identically zero for all times as $|x| \rightarrow \infty$.
} 
Explicitly, the macrotransport parameters $\bar{U}^{*}$ and $\bar{D}^{*}$ are computed by matching the asymptotic rates of change of the total moments of the microscale probability density,

$$
M_{m}\left(t \mid \phi_{\alpha}\right) \stackrel{\text { def. }}{=} \int_{-\infty}^{\infty} x^{m}\left(P_{a}+P_{b}\right) d x \quad(m=0,1,2, \ldots),
$$

against the corresponding moments,

$$
\bar{M}_{m}(t) \stackrel{\text { def. }}{=} \int_{-\infty}^{\infty} x^{m} \bar{P}(x, t) d x \quad(m=0,1,2, \ldots),
$$

of the macrotransport equation,

$$
\frac{\partial \bar{P}}{\partial t}+\bar{U}^{*} \frac{\partial \bar{P}}{\partial x}-\bar{D}^{*} \frac{\partial^{2} \bar{P}}{\partial x^{2}}=\delta(x) \delta(t) .
$$

In the latter, $\bar{P}$ is the so-called macrotransport probability density of the composite $a-b$ particle, governing the long-time asymptotic evolution of the true probability density, $P=P_{a}+P_{b}$. Solving equation (3.4) for $\bar{P}$ furnishes the gaussian distribution,

$$
\bar{P}=\left(4 \pi \bar{D}^{*} t\right)^{-1 / 2} \exp \left[-\frac{\left(x-\bar{U}^{*} t\right)^{2}}{4 \bar{D}^{*} t}\right] .
$$

With respect to the parameters appearing in eq. (2.1), application of the moment-matching scheme furnishes the composite Lagrangian velocity,

$$
\bar{U}^{*}=\frac{K_{b} U_{a}+K_{a} U_{b}}{K_{a}+K_{b}} \equiv \frac{K U_{a}+U_{b}}{1+K},
$$

where the parameter

$$
K \stackrel{\text { def. }}{=} \frac{K_{b}}{K_{a}}
$$

constitutes an equilibrium constant, representing the condition eventually achieved in eq. (2.1) when $K_{a} P_{a}=K_{b} P_{b}$, i.e. when the "forward" and "backward" rates at which the states change are in balance, at least in the global sense embodied in eq. (A.10). Consequently, the composite particle velocity (3.6) represents a weighted average of the respective state-specific velocities, $U_{\alpha}$, with the weighting corresponding to the stationary partitioning of states at long times. ${ }^{4}$ Albeit in a very different context, this result agrees with the comparable two-phase result of Aris (sans areal factors) [5], while also reducing to the results of Balakotaiah and Chang [2] upon setting one of the two state velocities to zero. Importantly, the mean velocity depends solely upon the stationary partitioning of the particle states, $K$, rather than being dependent upon the kinetic constant, $k$, governing the rate at which the particle transits between states before achieving this stationary partitioning of states.

\footnotetext{
${ }^{4}$ Substitution of the parameters appearing in our initial example 1.1 - 1.2 into eq. 3.6
} confirms that $\bar{U}^{*}=0$ in that case. 
The moment-matching scheme also furnishes the composite particle dispersivity,

$$
\bar{D}^{*}=\bar{D}^{M}+\bar{D}^{C}
$$

where

$$
\bar{D}^{M}=\frac{K_{b} D_{a}+K_{a} D_{b}}{K_{a}+K_{b}} \equiv \frac{K D_{a}+D_{b}}{1+K},
$$

represents the molecular or "Aris" contribution to the total dispersivity, and

$$
\bar{D}^{C}=\frac{K_{a} K_{b}}{\left(K_{a}+K_{b}\right)^{3}} \frac{\left(U_{a}-U_{b}\right)^{2}}{k} \equiv \frac{K}{(1+K)^{3}} \frac{\left(U_{a}-U_{b}\right)^{2}}{k K_{a}}
$$

constitutes the corresponding convective or "Taylor" dispersion. Of course, eqs. (3.8)-3.10) reduce appropriately to (1.7) in the special state-specific circumstances quantifying the latter. It is readily confirmed that $\bar{D}^{C}$ is positive semi-definite, vanishing only when: (i) the state velocities are identical in magnitude and direction; or (ii) one of the two parameters, $K_{\alpha}$, vanishes. The latter corresponds to the trivial transport process where, for long-times, the particle exists in but a single state. The functional form of eq. (3.10) is equivalent (to within a prefactor) to that derived by Aris [5]. Balakotaiah and Chang's result [2] is again recovered by setting one state velocity to zero.

At the present juncture, it is informative to compare the convective dispersivity, eq. (3.10), with the classical result 9 15] for the convective contribution to the Taylor-Aris dispersion of a solute entrained in a Poiseuille flow moving at mean velocity $\bar{v}$ through a cylindrical tube of radius $R$, namely

$$
\bar{D}^{C}=\frac{1}{48} \frac{\bar{v}^{2} R^{2}}{D}
$$

where $D$ is the molecular diffusivity of the solute. The quadratic dependence upon the velocity "inhomogeneity," $\left(U_{a}-U_{b}\right)^{2}$, appearing in (3.10) is analogous to the functional dependence, $\bar{v}^{2}$, appearing in (3.11). Moreover, the factor $k^{-1}$ appearing in (3.10) possesses an interpretation identical to the factor $R^{2} / D$ appearing in (3.11). Explicitly, each quantity respectively represents the characteristic time required for sampling the local space, either the "state" space in the present reactive problem or the physical space embodied in the tube's cross-sectional area, $\pi R^{2}$.

In contrast with $\bar{U}^{*}$, which is independent of the kinetic properties embodied in $k$, the convective dispersivity $\bar{D}^{C}$ depends upon both the particle's equilibrium and kinetic properties, respectively embodied in the parameters $K$ and $k$. The physical basis for this kinetic dependence may be rationalized by considering the transport of two particles, each occupying identical positions $x$ and states $\alpha$ at time $t$. Were both particles to remain in state $\alpha$, deviations in their relative mean positions would arise only from molecular diffusion, a phenomenon which is accounted for by the molecular contribution, $\bar{D}^{M}$, to the dispersivity, $\bar{D}^{*}$. In contrast, were the first particle to switch to state $\beta$ while the second remained in state $\alpha$, these two particles would tend to separate ("spread") due to the relative 
difference in their state-specific particle velocities, $\left|U_{\alpha}-U_{\beta}\right|$. Consider first the case where the reaction rate is rapid relative to the rate of convective spreading, i.e. $k \gg\left|U_{\alpha}-U_{\beta}\right| / \Delta x$, with $\Delta x$ a characteristic separation distance. [The combination $k(\Delta x)^{2}$ represents the coupling of interstate (local) transport to global transport, which is identical to the physical interpretation underlying $\bar{D}^{C}$.] For fast reactions, the likelihood is great that the particles will return to an identical state (either $\alpha$ or $\beta$ ), and hence identical velocity, in a brief period of time, thereby minimizing their "spreading." In contrast, for slow reactions, $k \ll\left|U_{\alpha}-U_{\beta}\right| / \Delta x$, there exists a high probability that the particles will travel significant distances before returning to identical states (and identical velocities), whereupon the "spreading" caused by the interstate transfer is expected to be much larger than in the fast reaction case. The appearance in $\bar{D}^{C}$ of the square, $\left(U_{a}-U_{b}\right)^{2}$, of the velocity difference rather than the term $\left|U_{a}-U_{b}\right|$ invoked in the preceding scaling arguments, arises from the fact that the dispersivity represents an asymptotic measure of the mean-squared deviation of the relative particle positions, rather than simply their absolute deviation.

An alternative rationalization of the dispersivity $\bar{D}^{*}$ is achieved by considering its role in quantifying the deviations of the instantaneous solute particle position, $x$, at time $t$ from its composite position, $\bar{U}^{*} t$, which the particle would occupy at time $t$ if it always moved uniformly at the velocity $\bar{U}^{*}$. To aid in this analysis, define the convected coordinate variable,

$$
x^{*} \stackrel{\text { def. }}{=} x-\bar{U}^{*} t
$$

Appropriate conversion of the microscale equation (2.1) into this altered coordinate format furnishes the following equation governing the respective $(a, b)$ transport processes:

$$
\frac{\partial P_{\alpha}}{\partial t}+\left(U_{\alpha}-\bar{U}^{*}\right) \frac{\partial P_{\alpha}}{\partial x^{*}}-D_{\alpha} \frac{\partial^{2} P_{\alpha}}{\partial x^{*^{2}}}+k\left(K_{\alpha} P_{\alpha}-K_{\beta} P_{\beta}\right)=\phi_{\alpha} \delta\left(x^{*}\right) \delta(t),
$$

where, here, $\partial / \partial t=(\partial / \partial t)_{x^{*}}$. In this new coordinate system, one of the two state-specific velocities takes place in the $+x^{*}$ direction, whereas the other occurs in the $-x^{*}$ direction (except for the trivial case where both state velocities are equal), since $\bar{U}^{*}$ represents an average of the respective state velocities, $U_{\alpha}$. Consequently, the transport process occurring in the convected $x^{*}$ coordinate system may be envisioned as a biased random walk, where the state-dependent step-sizes are proportional to the velocity difference, $\left|U_{\alpha}-\bar{U}^{*}\right|$, while the probability of taking a step in the $\alpha$-direction is proportional to $K_{\beta}$.

\section{Comparison with Numerical Results}

Numerical solutions are presented here for several illustrative choices of the transport and reaction parameters, comparing both the short- and long-time evolutions of the exact microscale probability density, $P=P_{a}+P_{b}$, with its

asymptotic counterpart, $\bar{P}$, given by eq. 3.5. For simplicity, we have eliminated intrastate molecular diffusion effects by setting $D_{a}=D_{b}=0$ in (3.13). 
Anticipating that, for long times, the total solute probability density will be convected at the rate $\bar{U}^{*}$, as in (3.6), we proceed with the formulation (3.13) of the microscale transport problem in the convected coordinate system, $x^{*}$. In rendering eq. (3.13) dimensionless, it proves convenient to abandon the egalitarian notation employed thus far, choosing state $a$ as the base state. ${ }^{5}$ With dimensionless time and length variables chosen as

$$
\tilde{t}=t k K_{a}, \quad \tilde{x}^{*}=x\left(\frac{k K_{a}}{U_{a}}\right),
$$

eq. (3.13) adopts the respective dimensionless forms

$$
\begin{aligned}
& \frac{\partial P_{a}}{\partial \tilde{t}}+\tilde{U}_{a} \frac{\partial P_{a}}{\partial \tilde{x}^{*}}+\left(P_{a}-K P_{b}\right)=\phi_{a} \delta\left(\tilde{x}^{*}\right) \delta(\tilde{t}), \\
& \cdot \frac{\partial P_{b}}{\partial \tilde{t}}+\tilde{U}_{b} \frac{\partial P_{b}}{\partial \tilde{x}^{*}}-\left(P_{a}-K P_{b}\right)=\left(1-\phi_{a}\right) \delta\left(\tilde{x}^{*}\right) \delta(\tilde{t}) .
\end{aligned}
$$

The equilibrium constant $K$ is given by (3.7), whereas the dimensionless statespecific velocities possess the respective forms

$$
\tilde{U}_{a}=\frac{1-\tilde{U}}{1+K}, \quad \tilde{U}_{b}=-K \tilde{U}_{a}
$$

with $\tilde{U}$ the velocity ratio,

$$
\tilde{U} \stackrel{\text { def. }}{=} \frac{U_{b}}{U_{a}} .
$$

In the convected, dimensionless coordinate system, our asymptotic result (3.5) adopts the form

$$
\bar{P}=\left(4 \pi \tilde{D}^{*} t\right)^{-1 / 2} \exp \left[-\frac{\left(\tilde{x}^{*}\right)^{2}}{4 \tilde{D}^{*} t}\right]
$$

with $\tilde{D}^{*}$, the dimensionless dispersivity,

$$
\tilde{D}^{*}=\frac{K(1-\tilde{U})^{2}}{(1+K)^{3}} .
$$

The coupled equation set (4.2)-(4.3) was solved numerically for various values of the parameters $K, \tilde{U}$ and $\phi_{a}$, using upwind finite differences for the advected terms and forward Euler time integration. Numerical results obtained for short $(\tilde{t}=0.5)$ and long $(\tilde{t}=50)$ times are depicted in Figs. 1 and 2, respectively, along with the corresponding asymptotic macrotransport results. Similar results (not depicted) were obtained for other choices of the parameters $K, \tilde{U}$ and $\phi_{a}$.

The results shown in Fig. 1 demonstrate that, for short-times, the system may exhibit dramatically different behavior from that displayed at longer times,

\footnotetext{
${ }^{5}$ Equivalent results may, of course, be obtained by choosing state $b$ as the base state and interchanging all subscripts.
} 
depending upon the particular choices made for the parameters $K$ and $\tilde{U}$ and initial state $\phi_{a}$ of the system. In general, the short-time transport of $a$ and $b$ as a whole is, not unexpectedly, bimodal, since that species whose velocity exceeds $\bar{U}^{*}$ moves in the $+\tilde{x}^{*}$ direction, while its counterpart moves in the $-\tilde{x}^{*}$ direction. Consequently, our asymptotic macrotransport analysis, which is symmetric about the origin $\tilde{x}^{*}=0$, invariably proves highly inaccurate when attempting to capture the short-time behavior, typically overestimating the width of the distribution. In contrast, as depicted in Fig. 2, the macrotransport description agrees very well with the numerical solutions for long-times for all choices of the parameters examined. As the species separate, the interstate reaction serves to "mix" their probability densities, ultimately giving rise to an asymptotically gaussian distribution.

It should be noted that the hyperbolic structure of the non-diffusive microscale equation (4.2)-(4.3) guarantees that, except for the special case $\tilde{U}_{a}=$ $\tilde{U}_{b}$, the exact solution of the microscale equations will be asymmetric with re-

spect to the origin. Explicitly, at time $\tilde{t}^{*}$, the maximum, $\tilde{x}_{\max }^{*}$, and minimum, $\tilde{x}_{\min }^{*}$, possible spatial positions with non-zero probability densities are given respectively by $\tilde{x}_{\max }^{*}=\tilde{U}_{a} \tilde{t}^{*}$ and $\tilde{x}_{\text {min }}^{*}=\tilde{U}_{b} \tilde{t}^{*}$ (taking $\tilde{U} \geq 1$ without any loss of generality). Aside from the aforementioned special case, the exact solution is expected to be asymmetric, since $\tilde{x}_{\max }^{*} \neq \tilde{x}_{\text {min }}^{*}$. In contrast, the macrotransport solution is not only symmetric about the origin, but also predicts a non-zero probability density for all values of $\tilde{x}^{*}$. However, this asymmetryinduced disparity existing between the exact solution and the macrotransport solution occurs in the tails of the probability density, at which positions the latter density is already exponentially small, and therefore of the order of the error incurred in our asymptotic analysis. In contrast, the differences between the exact and the macrotransport solutions are expected to be small in the "central region" for long times, as confirmed by the numerical results.

\section{Acknowledgments}

This work was supported in part by a Graduate Research Fellowship awarded to KDD by the National Science Foundation. We acknowledge useful discussions regarding the numerical solutions with Scott D. Phillips of MIT.

\section{A Moment-Matching Scheme}

Define the state-specific $m^{\text {th }}$-order "local" moment of the probability density of state $\alpha=(a, b)$ as

$$
P_{\alpha}^{(m)}\left(t \mid \phi_{\alpha}\right) \stackrel{\text { def. }}{=} \int_{-\infty}^{\infty} x^{m} P_{\alpha}\left(x, t \mid \phi_{\alpha}\right) d x \quad(m=0,1,2, \ldots) .
$$

The latter moments are equivalent to the local space (bounded) variables in conventional macrotransport theory [6]. In order to guarantee that these higher- 
order moments are finite, it is necessary to strengthen the attenuation condition (2.2), such that the probability densities and fluxes decay faster than algebraically, namely

$$
|x|^{m} P_{\alpha} \rightarrow 0 \quad \text { and } \quad|x|^{m} \partial P_{\alpha} / \partial x \rightarrow 0 \quad \text { as } \quad|x| \rightarrow \infty .
$$

The differential equation governing the state-specific local moment A.1 is derived as follows: (i) multiply eq. (2.1) by $x^{m}$; (ii) integrate over the range $-\infty<x<\infty$; (iii) integrate the resulting expression by parts; and (iv) subsequently apply the attenuation conditions (A.2). This scheme eventually furnishes the relation (for $\alpha \neq \beta$ )

$$
\begin{aligned}
\frac{d P_{\alpha}^{(m)}}{d t}+k\left[K_{\alpha} P_{\alpha}^{(m)}-K_{\beta} P_{\beta}^{(m)}\right]= & m U_{\alpha} P_{\alpha}^{(m-1)}+m(m-1) D_{\alpha} P_{\alpha}^{(m-2)}+ \\
& +\phi_{\alpha} \delta(t) \delta_{m 0},
\end{aligned}
$$

where $\delta_{m 0}$ is the Kronecker delta function (i.e. $\delta_{00}=1$ and $\delta_{m 0}=0$ for $m \neq 0$ ). In principle, this equation can be solved recursively for $P_{\alpha}^{(m)}\left(t \mid \phi_{\alpha}\right)$, although with use of macrotransport theory it proves unnecessary to do so in order to eventually calculate $\bar{U}^{*}$ and $\bar{D}^{*}$.

In a similar manner, the $m^{\text {th }}$-order "total moment" of the composite particle represents the sum of the corresponding local moments [cf. eq. (3.2)],

$$
M_{m}\left(t \mid \phi_{\alpha}\right)=P_{a}^{(m)}+P_{b}^{(m)} \quad(m=0,1,2, \ldots) .
$$

Summing eq. A.3 over the two states, $\alpha=(a, b)$, furnishes the following differential equation governing each $M_{m}$ :

$$
\begin{aligned}
\frac{d M_{m}}{d t}= & m\left[U_{a} P_{a}^{(m-1)}+U_{b} P_{b}^{(m-1)}\right]+m(m-1)\left[D_{a} P_{a}^{(m-2)}+D_{b} P_{b}^{(m-2)}\right]+ \\
& +\delta(t) \delta_{m 0} .
\end{aligned}
$$

The macrotransport parameters may then be computed from the following relationships [6]:

$$
\begin{aligned}
\bar{U}^{*} & =\lim _{t \rightarrow \infty} \frac{d M_{1}}{d t}, \\
\bar{D}^{*} & =\lim _{t \rightarrow \infty} \frac{d}{d t}\left(M_{2}-M_{1}^{2}\right),
\end{aligned}
$$

We proceed here to solve eqs. A.3 and (A.5), at least in the long-time limit (3.1). This enables us to establish the asymptotic forms of the moments, $P_{\alpha}^{(m)}$ and $M_{m}$, for $m=0,1,2$, thereby permitting $\bar{U}^{*}$ and $\bar{D}^{*}$ to be calculated via eqs. A.6)-A.7. Upon setting $m=0$ in eq. A.5 and integrating with respect to $t$, the zeroth-order total moment is found to be

$$
M_{0}= \begin{cases}0 & (t \leq 0) \\ 1 & (t>0)\end{cases}
$$


reflecting the conservation condition (2.3).

The zeroth-order, state-specific local moment equation is obtained by setting $m=0$ in eq. A.3, yielding

$$
\frac{d P_{\alpha}^{0}}{d t}+k\left(K_{\alpha} P_{\alpha}^{0}-K_{\beta} P_{\beta}^{0}\right)=\phi_{\alpha} \delta(t) \quad(\alpha \equiv a, b) .
$$

The asymptotic solution of this pair of equations, valid for times satisfying the inequality (3.1), is given by

$$
P_{\alpha}^{0} \approx P_{\alpha}^{0, \infty}+\exp , \quad P_{\alpha}^{0, \infty}=\frac{K_{\beta}}{K_{a}+K_{b}},
$$

where the symbol "exp" denotes position- and initial condition-dependent functions that are attenuated exponentially rapid in time. By forming the sum $P_{a}^{0, \infty}+P_{b}^{0, \infty}$ from eq. A.10, it is seen that the state-specific solutions A.10 asymptotically satisfy the normalization condition (2.3). Moreover, for long times, the local moments $P_{\alpha}^{0, \infty}$ are unconditional (rather than conditional) probability densities, being independent of both the initial position and the instantaneous state of the particle at $t=0$, as was to be expected.

The composite velocity $\bar{U}^{*}$ is computed by setting $m=1$ in eq. A.5, substituting the asymptotic solution A.10 for $\alpha=(a, b)$ into the resulting differential equation, and applying eq. (A.6), so as to obtain the result cited in eq. (3.6).

Subject to a posteriori verification, assume the following trial solutions for the first-order, state-specific local moments,

$$
P_{\alpha}^{1} \approx P_{\alpha}^{0, \infty}\left(\bar{U}^{*} t+B_{\alpha}\right)+\exp
$$

where the $B_{\alpha}$ are time-independent state-specific constants to be determined. Form the first moment of eq. A.3. with $m=1$, and use eq. A.10 for $\alpha=(a, b)$ together with the trial solution A.11 to obtain

$$
\bar{U}^{*}-U_{\alpha}=k K_{\alpha}\left(B_{\beta}-B_{\alpha}\right) .
$$

As in prior macrotransport analyses [6], the latter equation defines each $B_{\alpha}$ only to within a common, state-independent arbitrary constant, whose value proves irrelevant to the value of $\bar{D}^{*}$. Substituting eq. (3.6) into (A.12) furnishes the following relation between the $B_{\alpha}$ :

$$
B_{a}-B_{b}=\frac{U_{a}-U_{b}}{k\left(K_{a}+K_{b}\right)} .
$$

The latter time-independent solution, jointly with the fact that summing eq. (A.12) for $\alpha=(a, b)$ serves to reproduce eq. (3.6), constitutes a posteriori verification of the trial solution A.11). Substituting eqs. A.11) for $\alpha=(a, b)$ into eq. (A.4) with $m=1$ furnishes the first-order total moment,

$$
M_{1} \approx \bar{U}^{*} t+\frac{K_{b} B_{a}+K_{a} B_{b}}{K_{a}+K_{b}}+\exp ,
$$


which grows linearly in time at the rate $\bar{U}^{*}$.

Substitution of eqs. A.10 and A.11 for $\alpha=(a, b)$ into eq. A.5 with $m=2$ yields

$$
\frac{d M_{2}}{d t} \approx 2\left(\bar{U}^{*} \bar{U}^{*} t+\bar{D}^{M}+\frac{U_{a} K_{b} B_{a}+U_{b} K_{a} B_{b}}{K_{a}+K_{b}}\right)+\exp ,
$$

wherein $\bar{D}^{M}$ is given by eq. (3.9). Use of the latter equation, together with eqs. (A.7), (3.6), (A.13) and (A.14), furnishes the particle dispersivity (3.8).

\section{References}

[1] J. C. Giddings, H. Eyring, A molecular dynamic theory of chromatography, J. Phys. Chem. 59 (1955) 416-421.

[2] V. Balakotaiah, H. C. Chang, Dispersion of chemical solutes in chromatographs and reactors, Phil. Trans. Roy. Soc. A 351 (1995) 39-75.

[3] M. Gitterman, New applications of the two-state random model, Physica A 221 (1995) 330-339.

[4] F. Julicher, A. Ajdari, J. Prost, Modeling molecular motors, Rev. Mod. Phys. 69 (4) (1997) 1269-1281.

[5] R. Aris, On the dispersion of a solute by diffusion, convection and exchange between phases, Proc. Roy. Soc. London A 252 (1959) 67-77.

[6] H. Brenner, D. A. Edwards, Macrotransport Processes, ButterworthHeinemann, Boston, 1993.

[7] V. N. Kristensen, D. Kelefiotis, T. Kristensen, A.-L. Borrensen-Dale, High-throughput methods for detecting genetic variation, Biotechniques 30 (2001) 318-332.

[8] A. J. Nataraj, I. Olivos-Glander, N. Kusukawa, W. E. Highsmith Jr., Singlestrand conformation polymorphism and heteroduplex analysis for gel-based mutation detection, Electrophoresis 20 (1999) 1177-1185.

[9] G. I. Taylor, Dispersion of soluble matter in solvent flowing slowly through a tube, Proc. Roy. Soc. London A 219 (1953) 186-203.

[10] D. A. McQuarrie, Stochastic approach to chemical kinetics, J. Appl. Prob. 4 (1967) 413-478.

[11] D. T. Gillespie, Exact stochastic simulation of coupled chemical reactions, J. Phys. Chem. 81 (25) (1977) 2340-2361.

[12] M. Pagitsas, A. Nadim, H. Brenner, Multiple time scale analysis of macrotransport processes, Physica A 135A (1986) 533-550. 
[13] S. Haber, H. Brenner, Effect of entrained colloidal particles in enhancing the transport of adsorbable chemical contaminants, J. Colloid Interface Sci. 155 (1993) 226-246.

[14] G. Iosilevskii, H. Brenner, Taylor dispersion in discrete reactive mixtures, Chem. Eng. Comm. 133 (1995) 53-91.

[15] R. Aris, On the dispersion of a solute in a fluid flowing through a tube, Proc. Roy. Soc. London A 235 (1956) 67-77.

\section{Figure Captions}

Figure 1. Comparison between the numerical solution, $P$ (solid line), of the microscale equations and the solution, $\bar{P}$ (dashed line), of the macrotransport equation for the relatively short time $\tilde{t}=0.5$, and for four different choices of the equation parameters: (a) $K=1.5, \tilde{U}=-2.0, \phi_{a}=0.75$; (b) $K=2.0, \tilde{U}=0, \phi_{a}=0.25$; (c) $K=0.75, \tilde{U}=0.5, \phi_{a}=0.33$; (d) $K=0.5, \tilde{U}=-0.5, \phi_{a}=1$.

Figure 2. Comparison between the numerical solution, $P$ (solid line), of the microscale equations and the solution, $\bar{P}$ (dashed line), of the macrotransport equation for the relatively long time $\tilde{t}=50$, and for four different choices of the equation parameters: (a) $K=1.5, \tilde{U}=-2.0, \phi_{a}=0.75$; (b) $K=2.0, \tilde{U}=0, \phi_{a}=0.25$; (c) $K=0.75, \tilde{U}=0.5, \phi_{a}=0.33$; (d) $K=0.5, \tilde{U}=-0.5, \phi_{a}=1$. 


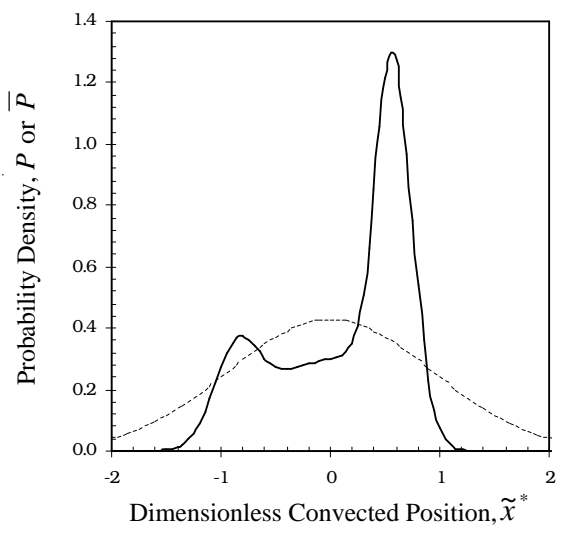

(a)

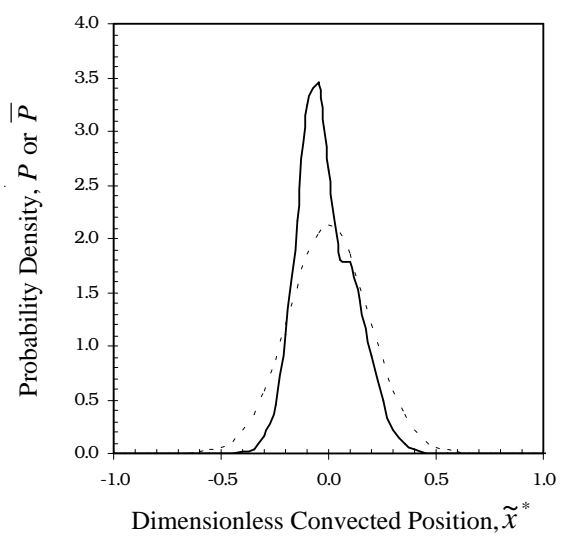

(c)

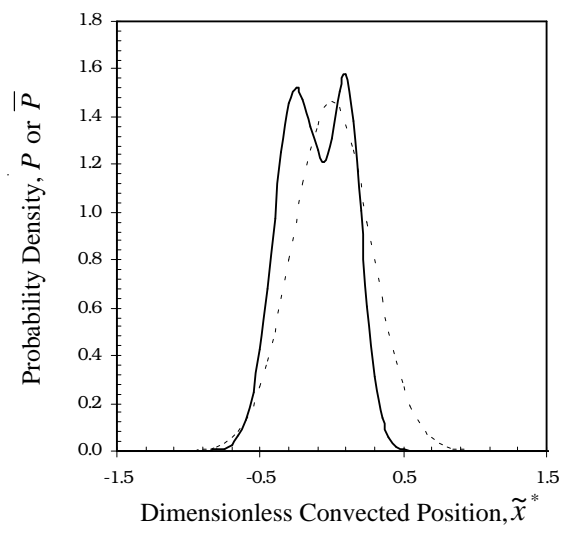

(b)

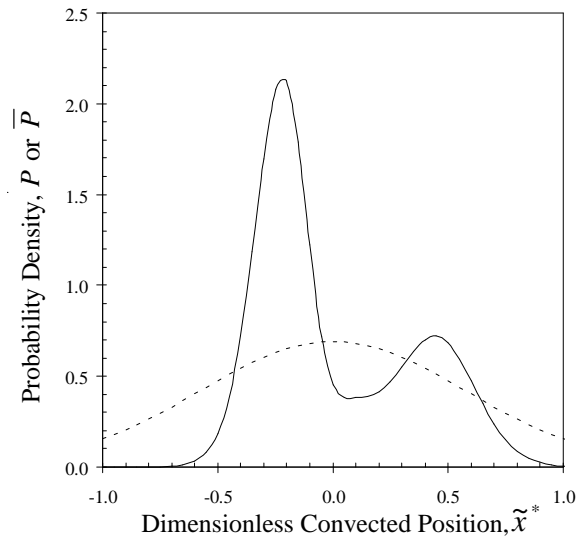

(d) 


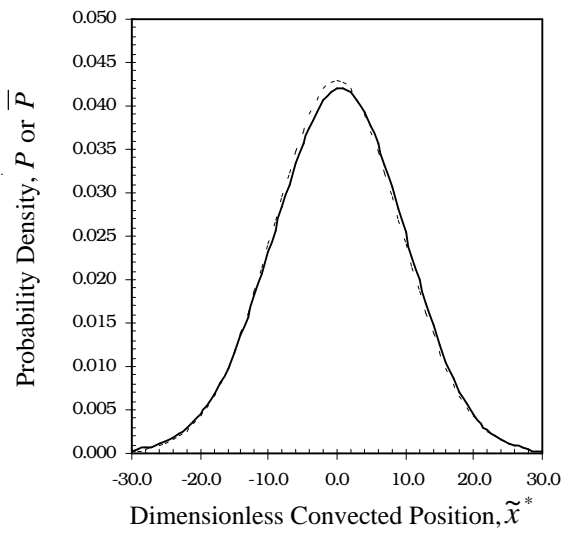

(a)

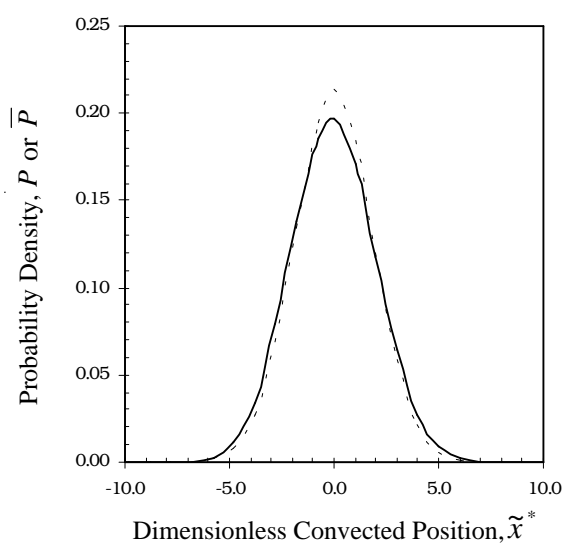

(c)

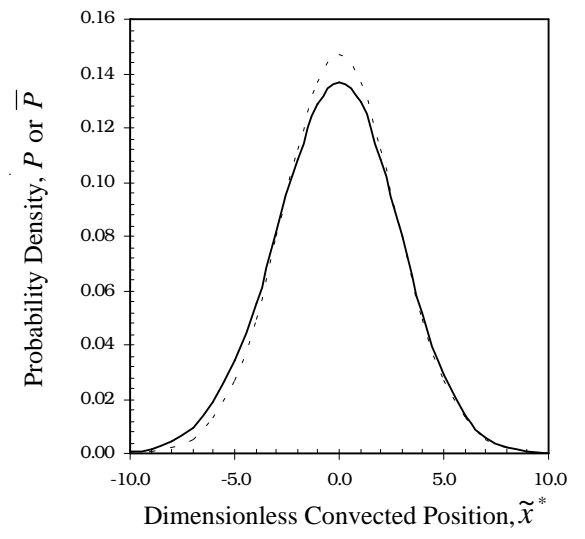

(b)

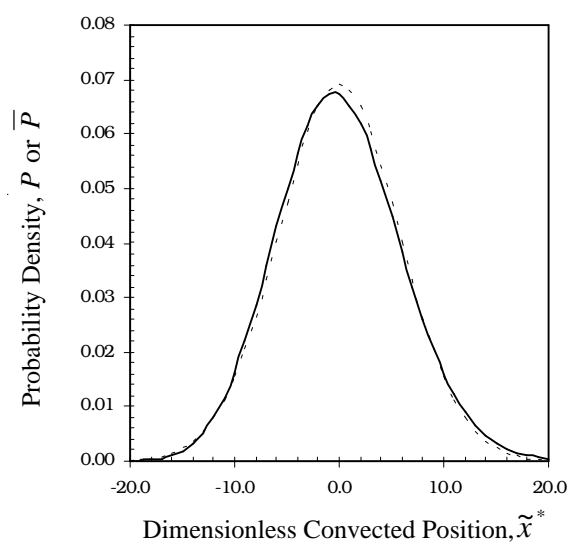

(d) 